\title{
PROGRAMA DE DETECCION Y CONTROL DE CANCER DE CUELLO UTERINO EN SERVICIO SALUD ARAUCANIA SUR*
}

\author{
Drs. Italo Capurro V., Juan A. Rojo E., Tulio Pino G., Claudio Vásquez Z., Jaime Garay \\ O., Sra. Mireya Venegas**
}

Servicio de Obstetricia y Ginecología, Unidad de Oncología Ginecológica. Hospital Regional de Temuco, Universidad de La Frontera

${ }^{* \star}$ Matrona

\section{RESUMEN}

Se analizan algunas características del Programa de Cáncer de Cuello Uterino en el SS Araucanía Sur. Se revisan los registros estadísticos de la Unidad de Oncología Ginecológica y de la Oficina de Informática del Servicio del Salud. Se aprecia que el cáncer de cuello uterino constituye el $68,19 \%$ de los cánceres ginecológicos, que la cobertura citológica promedio es de $56,2 \%$, la focalización citológica en grupo de 2564 años ha mejorado hasta un 89,67\%, la proporción CIS/Ca invasor se ha incrementado desde 0,12 a 1,35, las etapas precoces de invasión (Etapa I) ascendieron hasta el $62,5 \%$, la mortalidad ha tenido un descenso de $34 \%$ (14,45 x 100.000 en 1991, 9,46 x 100.000 en 2000). Subsisten problema de calidad de la muestra citológica, necesidad de aumentar su cobertura y bajar en forma aun más significativa la mortalidad, objetivo último del Programa.

\section{PALABRAS CLAVES: Carcinoma cérvico-uterino, programa de detección}

\section{SUMMARY}

Some characteristics of the Program for cervical carcinoma in southern Araucania Health Service, are analyzed. We reviewed the files of the Gynecological Oncology Unit and the Informatic Office; $68.19 \%$ of diagnosed Gynecological Carcinomas are Cancer of the Cervix uteri, the cytological coverage is $56.2 \%$, $89.67 \%$ of the cytologic smears are focused in the pre-established age risk group (25-64 yeras), the relation $\mathrm{Ca}$ in situ / invasive Cancer has improved from 0.12 to 1.35 ; the early stages of invasive carcinoma (Stage I) increased to $62.5 \%$, the mortality rate has decreased from $14.45 \times 100.000$ to $9.46 \times 100.000$ in 2000 . We have yet problems with the quality of smears, the need of increasing our cytological coverage and of course reduce more significatively the mortality rate, last objetive of the Program.

\section{KEY WORDS: Cervix uterus carcinoma, program for cervical cancer}

\section{INTRODUCCION}

El cáncer de cuello uterino presenta una alta incidencia y mortalidad en los países en desarrollo, encabezando la mayoría de los países latinoamericanos, entre ellos Chile, las estadísticas de letalidad por esta localización. Por las mismas razones anteriores, la importancia del cáncer de cuello en

*Trabajo recibido en abril de 2002 y aceptado para publicación por el Comité Editor en mayo de 2002. 
nuestro país y en nuestro Servicio es significativa. Como ha sido la regla, la neoplasia cervical maligna lidera los nuevos casos de cánceres ginecológicos pelvianos.

Chile, a nivel mundial, exhibe altas tasas de incidencia y mortalidad. En estadísticas de mortalidad por Ca de cuello uterino publicada en 1991 y referida al período 1984-1986, Chile aparece en tercer lugar después de Surinam y Costa Rica, con una tasa de mortalidad de 15,6 $\times 100$ mil mujeres (1). Aunque este índice ha experimentado una evidente disminución, llegando a una tasa promedio nacional de 9,6 × 100 mil mujeres (Minsal, 1988), aun fallecen en nuestro país 2 mujeres diarias por $\mathrm{Ca}$ de cérvicouterino, siendo la cuarta causa de muerte por cáncer en la mujer, luego del $\mathrm{Ca}$ de vesícula biliar, estómago y mama. En nuestra región constituye la tercera causa de muerte desplazando, al Ca de mama al cuarto lugar (2).

Hemos querido analizar en esta publicación, algunos aspectos del Programa de Detección Precoz de Cáncer de Cuello Uterino en el Servicio de Salud Araucanía Sur así como los logros y dificultades. Para el SS Araucanía Sur, la población femenina asignada mayor de 15 años es de 233.593 mujeres al año 2000 (Figura 1).

\section{MATERIAL Y METODO}

Se analiza el programa de Cáncer uterino del Servicio Araucanía Sur (que corresponde a la Provincia de Cautín en la IX Región de La Araucanía), con énfasis en lo ocurrido en los últimos 13 años. Desde 1974 este programa cubría la Región de la
Araucanía globalmente. En 1997 el SS de Salud se divide en SS Araucanía Norte y SS Araucanía Sur. Los datos presentados en esta ocasión, se refieren al SS Salud Araucanía Sur. Se revisaron los registros estadísticos de la Unidad de Oncología Ginecológica del Servicio de Obstetricia y Ginecología del Hospital Regional de Temuco y de la Oficina Informática Regional, en relación a la frecuencia relativa de los cánceres ginecológicos, al screening citológico en los aspectos de cobertura, calidad de la muestra y focalización etárea, así como, la proporción $\mathrm{CIS} / \mathrm{Ca}$ invasor diagnosticados, las Etapas Clínicas del $\mathrm{Ca}$ invasor, y la incidencia y mortalidad por cáncer de cuello. La Unidad de Oncología Ginecológica (UOG) del Hospital de Temuco, es el centro de referencia para la patología neoplásica ginecológica de la Región (SS Araucanía Sur y Norte).

\section{RESULTADOS}

La Tabla I muestra, en el período 1988-1998, la frecuencia relativa de los cánceres ginecológicos pelvianos, diagnosticados y tratados en nuestra Unidad de Oncología Ginecológica. De 1.110 cánceres ginecológicos, el $\mathrm{Ca}$ de cérvix uterino fue el más frecuente, constituyendo el $68,19 \%$ de los tumores malignos, seguido a distancia por el $\mathrm{Ca}$ de ovario con el $16,35 \%$.

En la Tabla II, se aprecia la cobertura citológica actual (año 2000) en el SS Araucanía Sur: con un promedio de $56,2 \%$ trianual con rangos para las diferentes comunas desde $97,6 \%$ a $34,9 \%$.

La Tabla III muestra la focalización etárea en los grupos de riesgo definido por MINSAL (25-64

Figura 1. IX Región de la Arauca-

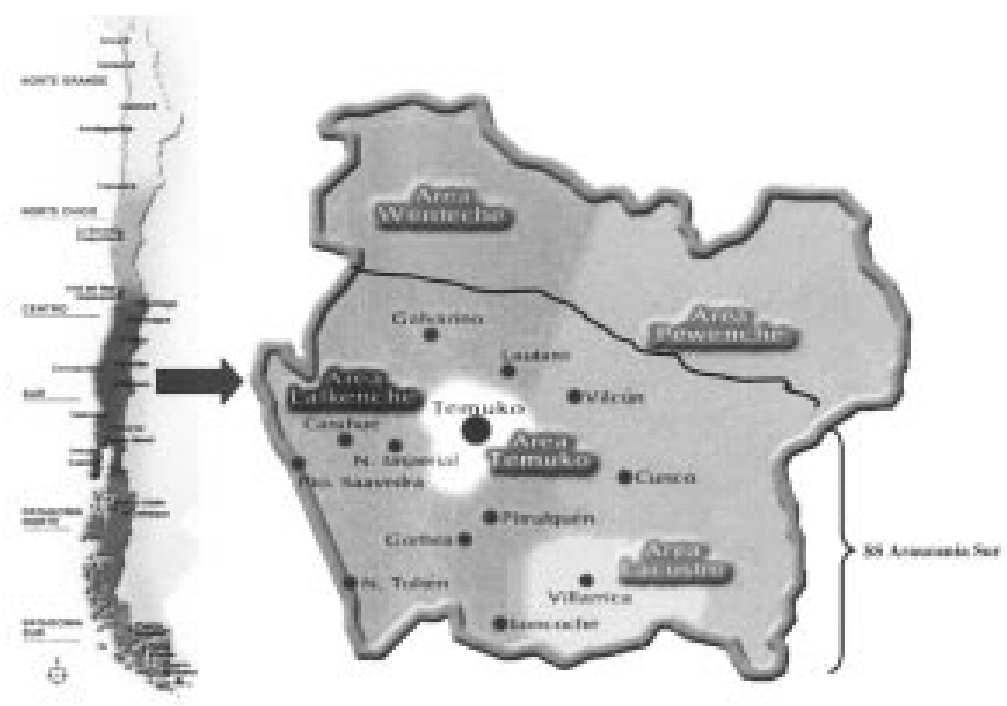
nía. S.S. Araucanía Sur. 
Tabla I

FRECUENCIA RELATIVA DE CANCERES GINECOLOGICOS*

\begin{tabular}{lrr}
\hline Localización & Casos & $\%$ \\
\hline Cuello uterino & 759 & 68,19 \\
Ovario & 182 & 16,35 \\
Endometrio & 98 & 8,80 \\
Vulva & 23 & 2,06 \\
Vagina & 19 & 1,70 \\
Trompa & 3 & 0,26 \\
Sarcomas & 26 & 2,33 \\
\hline Total & 1110 & \\
\hline
\end{tabular}

*Año 2000. Unidad Oncológica Ginecológica, Hospital de Temuco.

años): en 1997 un 77,05\% de las citologías se tomaban en ese tramo etáreo, cifra paulatinamente creciente hasta llegar a un $89,67 \%$ el año 2000.

La calidad de la toma de muestra citológica ha mantenido cifras aun insatisfactorias, con un $13,78 \%$ de frotis inadecuados en 1997 a un 10,95\% en 2000 , y un $21,07 \%$ para los frotis menos que óptimos en 1997 en relación a un 18,56\% el año 2000.

En las Tablas $\mathrm{V}$ y $\mathrm{VI}$ se destacan algunos indicadores de impacto temprano del programa:

Así, hay un progresivo y significativo aumento del diagnóstico del $\mathrm{Ca}$ invasor en etapas tempranas: La Etapa I constituía sólo el 10,9\% de nuestros cánceres invasores en 1987, llegando a superar el
Tabla II

\section{COBERTURA CITOLOGICA VIGENTE AÑO 2000 SS ARAUCANIA SUR*}

\begin{tabular}{lcc}
\hline Comuna & $\begin{array}{c}\text { Población } \\
\text { beneficiaria }\end{array}$ & $\begin{array}{c}\text { Cobertura } \\
\%\end{array}$ \\
\hline Temuco & 36,745 & 45,0 \\
Lautaro & 5.205 & 65,2 \\
Perquenco & 1.019 & 46,2 \\
Galvarino & 2.337 & 40,1 \\
Vilcún & 3.694 & 58,0 \\
Cunco & 3.271 & 66,6 \\
Imeperial & 6.540 & 56,0 \\
Carahue & 4.553 & 55,3 \\
Saavedra & 2.226 & 55,6 \\
Pitrufquén & 3.836 & 60,0 \\
Toltén & 1.684 & 56,0 \\
Gorbea & 2.739 & 65,9 \\
Loncoche & 4.480 & 63,6 \\
Villarrica & 7.015 & 58,0 \\
Pucón & 2.853 & 57,7 \\
Freire & 4.379 & 75,7 \\
Curarrehue & 918 & 93,6 \\
T. Schmidt & 2.448 & 97,6 \\
Melipeuco & 939 & 80,3 \\
P. Las Casas & 8.928 & 34,9 \\
\hline Total & 105.808 & 56,2 \\
\hline
\end{tabular}

*Población femenina 25 a 64 años.

$60 \%$ (62,5\% el año 2000). Asimismo han casi desaparecido las etapas ignoradas (Tabla V).

\section{Tabla III}

FOCALIZACION DE LA CITOLOGIA EN GRUPO DE RIESGO

\begin{tabular}{lrrrrrrrr}
\hline Grupo etáreo & \multicolumn{2}{c}{1997} & \multicolumn{2}{c}{1998} & \multicolumn{2}{c}{1999} & \multicolumn{2}{c}{2000} \\
\hline Menos de 25 años & 9.329 & $(21,23 \%)$ & 10.315 & $(24,59 \%)$ & 4.430 & $(10,52 \%)$ & 3.759 & $(8,31 \%)$ \\
$25-34$ años & 14.156 & $(32,22 \%)$ & 13.922 & $(33,19 \%)$ & 13.674 & $(32,48 \%)$ & 11.714 & $(25,89 \%)$ \\
$35-64$ años & 19.962 & $(44,83 \%)$ & 17.176 & $(40,95 \%)$ & 22.161 & $(52,63 \%)$ & 26.516 & $(58,62 \%)$ \\
Total 25-64 años & 33.848 & $(77,06 \%)$ & 31.898 & $(74,15 \%)$ & 35.835 & $(85,11 \%)$ & 40.558 & $(89,67 \%)$ \\
Más de 65 años & 747 & $(1,70 \%)$ & 525 & $(1,25 \%)$ & 1.837 & $(4,36 \%)$ & 1.351 & $(2,98 \% 0$ \\
\hline Total PAP & $43.924(100 \%)$ & $41.938(100 \%)$ & $42.102(100 \%)$ & 45.229 & $(100 \%)$ \\
\hline
\end{tabular}

Tabla IV

CALIDAD DE LA MUESTRA CITOLOGICA CERVICAL*

\begin{tabular}{lcrrr}
\hline & 1997 & 1998 & 1999 & 2000 \\
\hline Frotis inadecuado & $6.055(13,78 \%)$ & $5.015(11,95 \%)$ & $3.908(9,18 \%)$ & $4.954(10,95 \%)$ \\
Frotis menos que óptimo & $9.257(21,07 \%)$ & $7,89(18,81 \%)$ & $8.352(19,62 \%)$ & $8.395(18,56 \%)$ \\
\hline Total PAP & 43.429 & $41.936(100 \%)$ & $42.556(100,0 \%)$ & $45.229(100 \%)$ \\
\hline
\end{tabular}

*Región Araucanía (SS Araucanía Sur y Norte). 
Tabla V

DISTRIBUCION CANCER CERVICAL SEGUN ETAPAS CLINICAS*

\begin{tabular}{lcccccccccc}
\hline Etapa clínica & 1987 & 1990 & 1993 & 1994 & 1995 & 1996 & 1997 & 1998 & 1999 & 2000 \\
\hline I & 8 & 15 & 24 & 20 & 30 & 39 & 46 & 53 & 38 & 40 \\
& $10,9 \%$ & $25 \%$ & $29 \%$ & $38 \%$ & $40,1 \%$ & $59,1 \%$ & $56,8 \%$ & $51,5 \%$ & $60,3 \%$ & $62,5 \%$ \\
II & 24 & 22 & 21 & 13 & 19 & 11 & 16 & 22 & 9 & 16 \\
& $(32,87 \%)$ & $(40,0 \%)$ & $(25,6 \%)$ & $(25,0 \%)$ & $(25,6 \%)$ & $(16,66 \%)$ & $(19,8 \%)$ & $(21,4 \%)$ & $(14,28 \%)$ & $(25,0 \%)$ \\
III & 28 & 13 & 25 & 15 & 17 & 12 & 14 & 17 & 11 & 7 \\
& $(38,35 \%)$ & $(22,4 \%)$ & $(30,5 \%)$ & $(25,9 \%)$ & $(23,0 \%)$ & $(17,91 \%)$ & $(17,3 \%)$ & $(16,5 \%)$ & $(17,46 \%)$ & $(10,93 \%)$ \\
IV & 9 & 3 & 6 & 3 & 3 & 4 & 2 & 7 & 4 & 1 \\
& $(12,32 \%)$ & $(5,4 \%)$ & $(7,3 \%)$ & $(5,7 \%)$ & $(4,0 \%)$ & $(6,06 \%)$ & $(2,5 \%)$ & $(6,8 \%)$ & $(6,35 \%)$ & $(1,56 \%)$ \\
Ignorada & 4 & 2 & 6 & 1 & 5 & 1 & 3 & 4 & 1 & 0 \\
& $(5,48 \%)$ & $(3,63 \%)$ & $(7,3 \%)$ & $(1,9 \%)$ & $(6,7 \%)$ & $(1,51 \%)$ & $(3,7 \%)$ & $(3,9 \%)$ & $(1,58 \%)$ & $(0 \%)$ \\
Total casos & 73 & 58 & 82 & 52 & 74 & 67 & 81 & 103 & 63 & 64 \\
& $(100 \%)$ & $(100 \%)$ & $(100 \%)$ & $(100 \%)$ & $(100 \%)$ & $(100 \%)$ & $(100 \%)$ & $(100 \%)$ & $(100 \%)$ & $(100 \%)$ \\
\hline
\end{tabular}

*Región Araucanía (SS Araucanía Sur).

La Tabla VI muestra un sostenido ascenso de la proporción de $\mathrm{CIS} / \mathrm{Ca}$ invasor diagnosticados anualmente, desde 0,12 en 1993 a un 1,35 en el año 2001.

La tasa de mortalidad ha experimentado una reducción desde un $14,45 \times 100.000$ mujeres en 1991 a un $9,42 \times 100.000$ en 2000 (Tabla VII).

\section{DISCUSION}

La alta prevalencia del cáncer de cuello uterino, el conocimiento de su historia natural, el éxito del tratamiento de las lesiones preinvasoras, la aceptabilidad y el bajo costo de los métodos de detección de estas lesiones intraepiteliales mediante el

Tabla VI

PROPORCION CIS Y CANCER INVASOR 1991-2001 SS ARAUCANIA SUR

\begin{tabular}{lccc}
\hline Año & Ca in situ & Ca invasor & Proporción \\
\hline 1991 & 13 & 56 & 0,23 \\
1992 & 14 & 57 & 0,24 \\
1993 & 10 & 82 & 0,12 \\
1994 & 11 & 52 & 0,21 \\
1995 & 35 & 74 & 0,47 \\
1996 & 36 & 67 & 0,54 \\
1997 & 58 & 81 & 0,72 \\
1998 & 74 & 103 & 0,72 \\
1999 & 40 & 64 & 0,63 \\
2000 & 48 & 64 & 0,75 \\
2001 & 77 & 57 & 1,35 \\
\hline
\end{tabular}

screening citológico, han hecho posible y justificado, organizar Programas de Detección Precoz de esta neoplasia, los que logran un descenso significativo de la mortalidad específica, como ha sido demostrado en otros países como (Canadá, Columbia Británica) (3), países escandinavos (4).

El Programa de Detección de Cáncer de Cuello Uterino en nuestra Región, como parte del Programa Nacional, se inició en 1974. No ha estado exento de dificultades entre las que destacan la ruralidad de la Región $(43,1 \%)$ y los problemas sociales de marginalidad de la etnia mapuche que constituye el $30 \%$ de la población regional. A pesar de un número muy importante de lesiones intraepiteliales y microinva-

Tabla VI

VARIACION DE LA TASA DE MORTALIDAD POR CANCER CERVICOUTERINO SEGUN RESIDENCIA*

\begin{tabular}{cccc}
\hline Año & $\begin{array}{c}\text { Población } \\
\text { total }\end{array}$ & Casos & $\begin{array}{c}\text { Tasa } \times 100 \mathrm{mil} \\
\text { mujeres }\end{array}$ \\
\hline 1991 & 290.561 & 42 & 14,45 \\
1992 & 295.168 & 35 & 11,85 \\
1993 & 299.568 & 47 & 15,68 \\
1994 & 304.123 & 32 & 10,52 \\
1995 & 308.437 & 33 & 10,69 \\
1996 & 312.708 & 35 & 11,19 \\
1997 & 316.890 & 32 & 10,09 \\
1998 & 320.975 & 40 & 12,46 \\
1999 & 325.020 & 38 & 11,69 \\
2000 & 328.990 & 31 & 9,42 \\
\hline
\end{tabular}

*SS Araucanía Sur. Población femenina total. 
soras diagnosticadas y tratadas, la mortalidad por cuello uterino se mantuvo estable, como en el resto del país, por más de 15 años, con una tasa de alrededor del $15 \times 100.000$ mujeres de todas las edades.

La estrategia del Programa Nacional en los primeros 15 años, se basaba en la captación de las mujeres aprovechando sus canales habituales de motivación de consulta, como los controles de embarazo y planificación familiar, así como la consulta por morbilidad ginecológica. La frecuencia de toma citológica se efectuaba anualmente.

$\mathrm{Si}$ bien es cierto que se observaron algunos logros, algún efecto protector, como una reducción de la frecuencia relativa de los estadios invasores, un diagnóstico en etapas menos avanzadas y a edades más tempranas en las mujeres con antecedentes de PAP (5), la tasa de mortalidad se mantuvo, sin embargo, en las altas cifras iniciales, dado que la cobertura no llegó a superar el $15 \%$ anual.

Es sabido que para lograr un impacto significativo en la mortalidad, la cobertura citológica debe alcanzar al $80 \%$ de las mujeres en edad de riesgo.

En 1988 el Ministerio de Salud establece nuevas normas y estrategias para el Programa, entre las que destacan la definición de grupos de riesgo basados en la edad, para de esa manera focalizar los esfuerzos y cambia la frecuencia citológica a una toma trianual con el fin de optimizar los recursos y lograr un aumento significativo de la cobertura. Se favorece las edades en que más probablemente pesquisaremos (NIE de Alto grado o cánceres invasores muy iniciales. El Programa incluye entonces la toma de Pap a mujeres entre 25 y 64 años, cada tres años, privilegiando en primer lugar la mujer sin antecedentes de PAP y sobre 35 años, en segundo lugar la mujer con PAP atrasado y luego la mujer a la que le corresponde repetirse el PAP. Las mujeres de alto riesgo epidemiológico pueden hacer el PAP, aunque no estén en estos grupos etáreos, y lo deben repetir anualmente.

Luego de los 15 años, primeros años de aplicar las antiguas estrategias, no era fácil para los distintos involucrados, pacientes y equipo de salud, el cambio de conducta.

En nuestra Región, y sólo a partir de 1994 y luego de talleres con el equipo de salud, se logra un cambio, acorde a las nuevas normas del Ministerio. A partir de esa fecha se va alcanzando un aumento sostenido de la cobertura, a través de la implementación de estrategias educacionales, de captación y una mayor motivación del equipo de salud.

Aun cuando no se ha alcanzado la cobertura deseada mayor al $80 \%$ gracias a múltiples factores como el 56,2\% de cobertura actual (año 2000 en el SS Araucanía Sur, focalización mejorada, el aumento de la pesquisa del cáncer in situ, la mejoría de la gestión en términos del tiempo que demora el informe del PAP, el lapso de tiempo que media entre la atención y resolución de la paciente en el caso de las lesiones intraepiteliales, la continuidad de los Comités Oncológicos, la incorporación del Asa Electroquirúrgica en el arsenal terapéutico de las $\mathrm{NIE}$, la oportunidad del tratamiento quirúrgico y radioterapia de los cánceres invasivos, se ha logrado algunos avances que son significativos: disminución de la mortalidad, franca mejoría en la distribución del cáncer invasor por etapas clínica, atención en tiempos óptimos.

La cobertura anual en la región no pasaba de $15 \%$ al año 1989; el cambio de estrategia eleva la cobertura a $25 \%$ trianual. Desde 1994, cuando se manifiestan los efectos del cambio de actitud del equipo de salud hacia las nuevas estrategias del programa, se observa en forma sostenida un aumento de la cobertura trianual que llega en el año 2000 a un $56,2 \%$ para la Araucanía Sur.

Como vemos en la Tabla II, el incremento de cobertura es desigual en el SS Araucanía Sur, observándose los mayores índices en las comunas de Teodoro Schmidt y Curarrehue y los menores en las comunas de Temuco y Padre Las Casas, áreas predominantemente urbanas.

El Programa, en sus nuevas estrategias, debe focalizar mujeres entre 25 y 64 años, de modo que el número de citologías tomadas a mujeres de 25 años no alcance idealmente más del $5 \%$. Esta es también una cifra que paulatinamente ha ido mejorando, permitiendo que los recursos se concentren en los grupos etáreos más riesgosos (Tabla III). Desde el $24 \%$ de las citologías tomadas a mujeres menores de 25 años en 1998, se ha reducido al año 2000 a un $8,3 \%$.

La cobertura de mujeres mayores de 35 años, ha experimentado también una notoria mejoría llegando el 2000 a $54,3 \%$ en SS Araucanía Sur.

La adecuada toma de la muestra para el examen citológico que permita disminuir los falsos negativos del examen citológico, adquiere especial importancia dada la nueva frecuencia trianual del examen.

Nos preocupa por ello (Tabla IV) los porcentajes que aun se observan en citologías inadecuadas $(10,9 \%)$ y menos que óptima $(18,5 \%)$ en el año 2000 , que obliga a la repetición inmediata o al año respectivamente, lo que genera desvió de los recursos, en vez de aplicarse a nuevas pacientes.

Una cuidadosa ejecución de la toma de la muestra que debe ser representativa de la zona de 
transformación, así como reciclaje periódico en la capacitación deberán ser elementos que mejoren su calidad.

Los tiempos de latencia entre las distintas fases del proceso de pesquisa, diagnóstico y tratamiento son muy satisfactorios, ya que en un lapso de menos de tres meses desde la toma de la muestra, se completa el proceso de citación, estudio colposcópico e histológico y el correspondiente tratamiento.

Si bien el objetivo del programa es disminuir la incidencia y la mortalidad por cáncer cérvico invasor, hay algunos índices de impacto temprano del desarrollo de nuestro programa que son positivos: la distribución por etapas del carcinoma de cuello y la proporción de $\mathrm{Ca}$ in situ (cis) y cánceres invasores diagnosticados. En la Tabla V, vemos lo que sucedía en 1987, en 1990 y luego hasta el 2000. En 1987, sólo el 10,95\% de los nuevos casos se diagnosticaba en etapa I, en 1990 el $27,27 \%$ y en el año 2000 corresponde al $62 \%$, cambios que indudablemente serán un factor que contribuirá a la disminución de la mortalidad.

En lo que respecta a la proporción Cis/Ca invasor (Tabla VI), desde una preponderante presencia de Cis al inicio del programa (screening en una población virgen), paulatinamente la proporción fue descendiendo a favor del cáncer invasor como consecuencia de escrutar siempre la misma población "cautiva": (consultantes de Planificación Familiar y control prenatal), llegando al nivel más bajo en 1993 cuando la proporción fue de 0,12 , es decir se diagnosticaron 9 cánceres invasores por 1 cáncer in situ. Luego del cambio de estrategia, esta situación comenzó paulatinamente a revertirse, logrando llegar a 1,35 el año 2000 es decir diagnosticando casi 14 cáncer in situ por cada 10 cánceres invasores.

Una situación aceptable se constituye cuando se empieza a diagnosticar en mayor proporción cáncer in situ que cáncer invasor, que expresa un descenso de la incidencia de cáncer invasor, siendo la situación ideal diagnosticar por cada 3 cáncer in situ, uno invasor (proporción 3:1).

Como hemos señalado, la disminución de la tasa de Mortalidad por cáncer de cérvix uterino es el objetivo final del programa. Sabemos que reducciones significativas de este índice deben lograrse con coberturas citológicas superiores al $80 \%$. A pesar de ello en los últimos 10 años hemos tenido un descenso de un $34 \%$ en las tasas de mortalidad en el SS Araucanía Sur. Es así que en 1991, la tasa para Araucanía era de $14,4 \times 100.000$ mujeres, el año 1997 alcanza a 10,4 x 100,00 y el año 2000 a $9,4 \times 100,00$.
Creemos que en esta reducción han influido varios factores: el aumento de cobertura de 27 a $54 \%$ (aumento que sabemos aun insuficiente) gracias a la motivación particular del equipo de salud involucrado. Este aumento ha sido posible también por el esfuerzo de nuestro Laboratorio Regional de citopatología que ha enfrentado, sin aumento significativo de los recursos, la mayor demanda por citología, incluyendo la implementación de estrictos criterios de control de calidad internos y externos. A esto se ha agregado la oportunidad y acortamiento de los tiempos en la atención de las pacientes con lesiones intraepiteliales e invasoras. La posibilidad de hacer radioterapia en Temuco, los pabellones oportunos, el funcionamiento del Comité OncoGinecológico, la mejoría en el seguimiento de las pacientes con cáncer invasor, han sido otros factores importantes que han contribuido al descenso de la mortalidad.

Sin dudas estos son avances importantes, pero aun estamos lejos de las cifras de mortalidad de países desarrollados: Canadá, tasa de 2,3 x 100 mil, USA e Islandia 2,5 x 100 mil, cuyos Programas de screening han sido exitosos (6). Nuestra cobertura citológica debe alcanzar en un plazo razonable al menos el $80 \%$; para ello es necesario destinar recursos en el área de la captación de las muestras, en el aumento de la capacidad diagnóstica del Laboratorio de Citopatología, así como en la Unidad de Oncología Ginecológica con implementación y pabellones que permitan hacer frente exitosamente al aumento de la pesquisa de lesiones invasoras e intraepiteliales.

Es importante acrecentar también los recursos en el campo de la educación o del trabajo en el área de las ciencias sociales, para hacer un efectivo trabajo comunitario y lograr que la mujer internalice la necesidad de su propia preocupación por la salud.

De esta manera podremos continuar descendiendo nuestras tasas de mortalidad a un nivel mucho más significativo, para beneficio de nuestras pacientes y de sus familias.

Estas dificultades deberán ser resueltas por todos los actores involucrados: autoridades de salud, equipo de salud y las propias interesadas conscientes del autocuidado en salud.

\section{BIBLIOGRAFIA}

1. Boring CC, Squires TS, Tong T: Fifteen countries with the highest mortality rates for cancer of the cervix, 1984-1986. Cancer Statistics CA. Cancer J Clin 1991; 41: 19. 
2. Minsal Chile: Anuario de Defunciones y Causas de Muerte, 1988.

3. Anderson George H, Boyes David A, Benedet $\mathrm{JL}$ et al: Organisation and results of the cervical cytology screening programme in British Columbia, 1955-85. Br Med J 1988; 296-975-78.

4. Laara E, Day Nicholas E, Hakama Matti: Trends in mortality from cervical cance in the nordic countries: association with organized screening programmes. Lancet 1987; 1: 1247-49.

5. Capurro I, Rojo JA, Jorquera J, Dabancens A: Efecos inmediatos del Programa de control de cáncer cervicouterino sobre la mortalidad en la IX Región de la Araucanía. Rev Chil Obstet Ginecol 1988; 53: 321.

6. Wingo PA, Tong T, Bolden S: Cancer statistics 1995 CA; Cancer J Clin 1995; 45(1): 8-30. 